\title{
Enzymatic Removal of Carboxyl Protecting Groups. Part II: Cleavage of the Benzyl and Methyl Moieties
}

\author{
Efrosini Barbayianni, ${ }^{\S, \dagger}$ Irene Fotakopoulou, ${ }^{\ddagger}, \dagger$ Marlen Schmidt, ${ }^{¥}$ \\ Violetta Constantinou-Kokotou, ${ }^{\ddagger}$ Uwe T. Bornscheuer, ${ }^{\sharp *}$ \\ George Kokotos ${ }^{\S, *}$ \\ ${ }^{\S}$ Laboratory of Organic Chemistry, Department of Chemistry, University of Athens, \\ Panepistimiopolis, Athens 15771, Greece \\ ${ }^{\ddagger}$ Chemical Laboratories, Agricultural University of Athens, Iera Odos 75, Athens 11855 , \\ Greece \\ ${ }^{¥}$ Department of Technical Chemistry and Biotechnology, Institute of Chemistry and \\ Biochemistry, Greifswald University, Soldmannstr. 16, D-17487 Greifswald, Germany
}

gkokotos@cc.uoa.gr

uwe.bornscheuer@uni-greifswald.de

Supporting Information

Table of contents

Experimental section

Synthesis of substrates

Products of enzymatic hydrolysis 


\section{EXPERIMENTAL SECTION}

Melting points were determined on a Buchi apparatus and are uncorrected. Specific rotations were measured at $25^{\circ} \mathrm{C}$ on a Perkin-Elmer 343 polarimeter using a $10 \mathrm{~cm}$ cell. NMR spectra were recorded on $200 \mathrm{MHz}$ spectrometer. All amino acid derivatives were purchased from Fluka and Bachem. TLC plates (silica gel 60 F254) and silica gel 60 (70-230 or 230-400 mesh) for column chromatography were purchased from Merck. Visualization of spots was effected with UV light and/or phosphomolybdic acid and/or ninhydrin, both in EtOH stain. Esterase BsubpNBE was produced recombinantly (see main text), but is also available from Jülich Fine Chemicals (Esterase BS2, www.juelich-chemicals.com, Jülich, Germany). 50 mg of commercial available CAL-A correspond to 1830 Units (manufactures instructions (Roche Diagnostics): $>30 \mathrm{U} / \mathrm{mg}_{\mathrm{Lyo}}$ towards tributyrin as substrate). $50 \mathrm{mg}$ lyophilisate of recombinant BsubpNBE / BS2 correspond to $20 \mathrm{mg}$ protein or $7.5 \mathrm{mg}$ BsubpNBE or 600 Units with $p$ nitrophenyl acetate (pNPA) as substrate (80U/mg protein $\left._{\text {towards }} \mathrm{pNPA}\right)$.

\section{Synthesis of substrates}

\section{General method for the preparation of benzyl esters}

To a stirred solution of the acid $(1 \mathrm{mmol})$ and benzyl alcohol $(0.3 \mathrm{~mL}, 3 \mathrm{mmol})$ in $\mathrm{CH}_{2} \mathrm{Cl}_{2}(2$ $\mathrm{mL})$, 4-(dimethylamino)pyridine $(0.01 \mathrm{~g}, \quad 0.1 \mathrm{mmol})$ and subsequently $N, N^{\prime}-$ dicyclohexylcarbodiimide $(0.25 \mathrm{~g}, 1.2 \mathrm{mmol})$ were added at $0^{\circ} \mathrm{C}$. The reaction mixture was stirred for $1 \mathrm{~h}$ at $0^{\circ} \mathrm{C}$ and overnight at room temperature. After filtration, the solvent was evaporated under reduced pressure and EtOAc $(20 \mathrm{~mL})$ was added. The organic layer was washed consecutively with brine, $1 \mathrm{~N} \mathrm{HCl}$, brine, $5 \% \mathrm{NaHCO}_{3}$, and brine, dried over $\mathrm{Na}_{2} \mathrm{SO}_{4}$ and evaporated under reduced pressure. The residue was purified by column chromatography using $\mathrm{CHCl}_{3}$ as eluent. 


\section{Benzyl 4-(benzyloxycarbonyl)butanoate (11, Table 1)}

Yield 51\%; Oil; ${ }^{1} \mathrm{H}$ NMR (200 MHz, $\left.\mathrm{CDCl}_{3}\right): \delta 7.44-7.26(\mathrm{~m}, 10 \mathrm{H}), 5.12(\mathrm{~s}, 2 \mathrm{H}), 5.10(2 \mathrm{H}$, $\mathrm{s}), 4.91(\mathrm{~m}, 1 \mathrm{H}), 3.25(\mathrm{~m}, 2 \mathrm{H}), 2.42(\mathrm{t}, 2 \mathrm{H}, J=7.4 \mathrm{~Hz}), 1.86(\mathrm{~m}, 2 \mathrm{H}) ;{ }^{13} \mathrm{C} \mathrm{NMR}(50 \mathrm{MHz}$, $\left.\mathrm{CDCl}_{3}\right): \delta 173.0,156.4,136.4,135.7,128.5,128.4,128.3,128.2,128.1,127.5,126.9,66.6$, 66.3, 40.3, 31.4, 25.0; Anal. Calcd for $\mathrm{C}_{19} \mathrm{H}_{21} \mathrm{NO}_{4}$ : C, 69.71; H, 6.47; N, 4.28. Found: C, 69.55; H, 6.53; N, 4.34.

\section{(S)-1-benzyl 5-tert-butyl 2-(benzyloxycarbonyl)pentanedioate (17, Table 1)}

Yield 71\%; Oil; $[\alpha]_{\mathrm{D}}=-0.6\left(c 1, \mathrm{CHCl}_{3}\right) ;{ }^{1} \mathrm{H} \mathrm{NMR}\left(200 \mathrm{MHz}, \mathrm{CDCl}_{3}\right): \delta 7.36-7.31(\mathrm{~m}, 10 \mathrm{H})$, $5.46(\mathrm{~d}, 1 \mathrm{H}, J=7.2 \mathrm{~Hz}), 5.18(\mathrm{~s}, 2 \mathrm{H}), 5.12(\mathrm{~s}, 2 \mathrm{H}), 4.45(\mathrm{~m}, 1 \mathrm{H}), 2.29(\mathrm{~m}, 2 \mathrm{H}), 2.20(\mathrm{~m}, 1 \mathrm{H})$ $1.98(\mathrm{~m}, 1 \mathrm{H}), 1.43$ (br s, 9H); ${ }^{13} \mathrm{C} \mathrm{NMR}\left(50 \mathrm{MHz}, \mathrm{CDCl}_{3}\right): \delta 173.0,171.8,155.9,136.1$, 135.2, 128.6, 128.5, 128.4, 128.2, 128.1, 128.0, 80.8, 67.2, 67.0, 53.5, 31.3, 28.0, 27.5; Anal. Calcd for $\mathrm{C}_{24} \mathrm{H}_{29} \mathrm{NO}_{6}$ : C, 67.43; H, 6.84; N, 3.28. Found: C, 67.49; H, 7.05; N, 3.15.

Substrates 2, 4, 7, 10 and 15 (Table 1) were prepared according to the procedure for benzyl esters described above. The analytical data were in accordance with those reported in the literature (substrate $\mathbf{2}^{1}$, substrate $\mathbf{4}^{2}$, substrate $\mathbf{7}^{3}$, substrate $\mathbf{1 0}{ }^{4}$, substrate $\mathbf{1 5}^{5,6}$ ).

\section{Methyl 4-(tert-butoxycarbonyl)butanoate (8, Table 1)}

To a stirred solution of HCl.H-GABA-OMe (0.31 g, $2 \mathrm{mmol})$ in $\mathrm{MeOH}(18 \mathrm{~mL}), \mathrm{Et}_{3} \mathrm{~N}(2 \mathrm{~mL}$, $14.4 \mathrm{mmol})$ and subsequently $\mathrm{Boc}_{2} \mathrm{O}(0.66 \mathrm{~g}, 3 \mathrm{mmol})$ were added. The reaction mixture was stirred for $1 \mathrm{~h}$ at room temperature. $\mathrm{MeOH}$ was evaporated under reduced pressure and the residue was purified by column chromatography using $\mathrm{CHCl}_{3}$ as eluent. 
Yield $0.27 \mathrm{~g}(61 \%)$; white solid; mp $47-49^{\circ} \mathrm{C} ;{ }^{1} \mathrm{H}$ NMR $\left(200 \mathrm{MHz}, \mathrm{CDCl}_{3}\right): \delta 4.69(\mathrm{~m}, 1 \mathrm{H})$, $3.66(\mathrm{~s}, 3 \mathrm{H}), 3.14(\mathrm{~m}, 2 \mathrm{H}), 2.35(\mathrm{t}, 2 \mathrm{H}, J=7.4 \mathrm{~Hz}), 1.80(\mathrm{~m}, 2 \mathrm{H}), 1.42[\mathrm{br} \mathrm{s}, 9 \mathrm{H}] ;{ }^{13} \mathrm{C} \mathrm{NMR}$ $\left(50 \mathrm{MHz}, \mathrm{CDCl}_{3}\right): \delta 173.7,155.9,79.2,51.6,39.8,31.2,28.3,25.2$; Anal. Calcd for $\mathrm{C}_{10} \mathrm{H}_{19} \mathrm{NO}_{4}$ : C, 55.28; H, 8.81; N, 6.45. Found: C, 55.45; H, 8.67; N, 6.48.

\section{Methyl 4-(benzyloxycarbonyl)butanoate (9, Table 1)}

To a stirred solution of HCl.H-GABA-OMe $(0.31 \mathrm{~g}, 2 \mathrm{mmol})$ in 1,4-dioxane $(3.6 \mathrm{~mL})$, a $2 \mathrm{M}$ aqueous solution of $\mathrm{Na}_{2} \mathrm{CO}_{3}(2.2 \mathrm{~mL}, 4.4 \mathrm{mmol})$ was added, followed by benzyl chloroformate $(0.3 \mathrm{~mL}, 2.1 \mathrm{mmol})$. The mixture was stirred at room temperature for $4 \mathrm{~h}$ and then partitioned between ethyl acetate and water. The organic layer was washed with brine, dried over $\mathrm{Na}_{2} \mathrm{SO}_{4}$ and evaporated under reduced pressure. The residue was purified by column chromatography using $\mathrm{CHCl}_{3}$ as eluent.

Yield 0.33 g (66\%); Oil; ${ }^{1} \mathrm{H}$ NMR (200 MHz, $\left.\mathrm{CDCl}_{3}\right): \delta 7.32(\mathrm{~m}, 5 \mathrm{H}), 5.20(\mathrm{~m}, 1 \mathrm{H}), 5.07$ (s, 2H), $3.63(\mathrm{~s}, 3 \mathrm{H}), 3.20(\mathrm{~m}, 2 \mathrm{H}), 2.34(\mathrm{t}, 2 \mathrm{H}, J=7.4 \mathrm{~Hz}), 1.81(\mathrm{~m}, 2 \mathrm{H}) ;{ }^{13} \mathrm{C}$ NMR $(50 \mathrm{MHz}$, $\left.\mathrm{CDCl}_{3}\right): \delta 173.5,156.3,136.4,128.3,127.9,66.4,51.5,40.1,31.0,24.9$; Anal. Calcd for $\mathrm{C}_{13} \mathrm{H}_{17} \mathrm{NO}_{4}$ : C, 62.14; H, 6.82; N, 5.57. Found: C, 62.38; H, 6.75; N, 5.76.

\section{Benzyl 4-(((9H-fluoren-9-yl)methoxy)carbonyl)butanoate (13, Table 1)}

To a stirred suspension of Fmoc-GABA-OH $(0.5 \mathrm{~g}, 1.54 \mathrm{mmol})$ in benzene $(5 \mathrm{~mL})$, benzyl alcohol (1.0 g, $9.24 \mathrm{mmol})$ and $p$-toluenesulfonic acid $(0.08 \mathrm{~g}, 0.46 \mathrm{mmol})$ were added. The reaction mixture was stirred under reflux for $48 \mathrm{~h}$. The homogeneous solution was cooled down, washed with water, $5 \% \mathrm{NaHCO}_{3}$, dried over $\mathrm{Na}_{2} \mathrm{SO}_{4}$ and the organic solvent was evaporated under reduced pressure. The residue was purified by column chromatography using Petroleum ether $\left(60-80^{\circ} \mathrm{C}\right) /$ EtOAc $(6: 4)$ as eluent. 
Yield 0.46 g (72\%); oil; ${ }^{1} \mathrm{H}$ NMR $\left(200 \mathrm{MHz}, \mathrm{CDCl}_{3}\right): \delta 7.76(\mathrm{~d}, 2 \mathrm{H}, J=6.8 \mathrm{~Hz}), 7.58(\mathrm{~d}, 2 \mathrm{H}$, $J=7 \mathrm{~Hz}), 7.35(\mathrm{~m}, 9 \mathrm{H}), 5.12(\mathrm{~s}, 2 \mathrm{H}), 4.92(\mathrm{~m}, 1 \mathrm{H}), 4.40(\mathrm{~d}, 2 \mathrm{H}, J=6.6 \mathrm{~Hz}), 4.20(\mathrm{t}, 2 \mathrm{H}, J=$ $6.8 \mathrm{~Hz},), 3.23(\mathrm{~m}, 2 \mathrm{H}), 2.40(\mathrm{t}, 2 \mathrm{H}, J=7.4 \mathrm{~Hz}), 1.85(\mathrm{~m}, 2 \mathrm{H}) ;{ }^{13} \mathrm{C} \mathrm{NMR}\left(50 \mathrm{MHz}, \mathrm{CDCl}_{3}\right): \delta$ $173.0,156.4,143.9,141.3,135.8,128.6,128.5,128.3,128.2,127.62,127.56,127.0,126.9$, 124.9, 119.9, 66.5, 66.4, 47.2, 40.3, 31.4, 25.1; Anal. Calcd for $\mathrm{C}_{26} \mathrm{H}_{25} \mathrm{NO}_{4}$ : C, 75.16; $\mathrm{H}, 6.06$; N, 3.37. Found: C, 75.32; H, 6.17; N, 3.18.

\section{Methyl 4-(((9H-fluoren-9-yl)methoxy)carbonyl)butanoate (12, Table 1)}

To a stirred solution of HCl.H-GABA-OMe (0.50 g, $4.3 \mathrm{mmol})$ in 1,4-dioxane $(4.5 \mathrm{~mL})$, a $10 \%$ aqueous solution of $\mathrm{Na}_{2} \mathrm{CO}_{3}(11 \mathrm{~mL}, 10.4 \mathrm{mmol})$ was added, followed by 9fluorenylmethyl chloroformate $(1.16 \mathrm{~g}, 4.5 \mathrm{mmol})$. The mixture was stirred overnight at room temperature and then acidified until $\mathrm{pH}$ 2. The organic layer was washed with brine, dried over $\mathrm{Na}_{2} \mathrm{SO}_{4}$ and evaporated under reduced pressure. The residue was purified by column chromatography using $\mathrm{CHCl}_{3}$ as eluent.

Yield $0.67 \mathrm{~g}(46 \%)$; White solid; mp 98-100 ${ }^{\circ} \mathrm{C} ;{ }^{1} \mathrm{H}$ NMR $\left(200 \mathrm{MHz}, \mathrm{CDCl}_{3}\right): \delta 7.69(\mathrm{~d}, 2 \mathrm{H}, J$ $=7.6 \mathrm{~Hz}), 7.52(\mathrm{~d}, 2 \mathrm{H}, J=7.6 \mathrm{~Hz}), 7.29(\mathrm{~m}, 4 \mathrm{H}), 4.92(\mathrm{~m}, 1 \mathrm{H}), 4.33(\mathrm{~d}, 2 \mathrm{H}, J=6.6 \mathrm{~Hz}), 4.14$ $(\mathrm{t}, 1 \mathrm{H}, J=6.6 \mathrm{~Hz}), 3.60(\mathrm{~s}, 3 \mathrm{H}), 3.15(\mathrm{~m}, 2 \mathrm{H}), 2.29(\mathrm{t}, 2 \mathrm{H}, J=7.4 \mathrm{~Hz}), 1.77(\mathrm{~m}, 2 \mathrm{H}) ;{ }^{13} \mathrm{C}$ NMR (50 MHz, $\left.\mathrm{CDCl}_{3}\right): \delta 173.6,156.4,143.9,141.2,127.6,126.9,124.9,119.9,66.5,51.6$, 47.2, 40.3, 31.1, 25.0; Anal. Calcd for $\mathrm{C}_{20} \mathrm{H}_{21} \mathrm{NO}_{4}$ : C, 70.78; H, 6.24; N, 4.13. Found: $\mathrm{C}$, $70.59 ; \mathrm{H}, 6.28 ; \mathrm{N}, 4.26$.

Substrates $\mathbf{1}^{7,8}, \mathbf{3}^{7,2}, \mathbf{1 4}^{9}$ and $\mathbf{1 6}^{10}$ (Table 1 ) were synthesized according to the literature. 
Dipeptides 1, 2 and 3 (Table 2) were synthesized by the WSCI/HOBt method. ${ }^{11}$ Their analytical data were in accordance with those reported in the literature (substrate $\mathbf{1}^{12}$, substrate $\mathbf{2}^{13}$, substrate $\left.\mathbf{3}^{14}\right)$.

Substrate 4 (Table 2) was prepared according to an already known procedure. ${ }^{15}$

\section{Products of enzymatic hydrolysis}

\section{(S)-2-((S)-2-(benzyloxycarbonyl)-3-phenylpropanamido)-4-methylpentanoic acid}

White solid; mp $126-128^{\circ} \mathrm{C} ;[\alpha]_{\mathrm{D}}=-13.0\left(c 1, \mathrm{CH}_{3} \mathrm{OH}\right) ;{ }^{1} \mathrm{H}$ NMR $\left(200 \mathrm{MHz}, \mathrm{CDCl}_{3}\right): \delta 8.39$ (br s, 1H), 7.33-7.15 (m, 10H), $6.60(\mathrm{~d}, 1 \mathrm{H}, J=7 \mathrm{~Hz}), 5.71(\mathrm{~d}, 1 \mathrm{H}, J=7.2 \mathrm{~Hz}), 5.02(\mathrm{~s}, 2 \mathrm{H})$, $4.51(\mathrm{~m}, 2 \mathrm{H}), 3.03(\mathrm{~d}, 2 \mathrm{H}, J=6.6 \mathrm{~Hz}), 1.54(\mathrm{~m}, 3 \mathrm{H}), 0.85(\mathrm{~d}, 6 \mathrm{H}, J=5.4 \mathrm{~Hz}) ;{ }^{13} \mathrm{C}$ NMR $(50$ $\left.\mathrm{MHz}, \mathrm{CDCl}_{3}\right): \delta 176.0,171.5,156.3,136.2,136.0,129.3,128.6,128.5,128.2,127.9,127.0$, 67.2, 56.1, 50.9, 40.9, 38.2, 24.7, 22.7, 21.8; Anal. Calcd for $\mathrm{C}_{23} \mathrm{H}_{28} \mathrm{~N}_{2} \mathrm{O}_{5}$ : C, 66.97; H, 6.84; N, 6.79. Found: C, 66.84; H, 6.89; N, 6.83.

The other products of the enzymatic hydrolysis were identified by their analytical data in comparison with authentic samples.

\section{REFERENCES}

(1) Roy, O.; Riahi, A.; Hénin, F.; Muzart, J. Eur. J. Org. Chem. 2002, 3986-3994.

(2) Crosignani, S.; White, P. D.; Steinauer, R.; Linclau, B. Org. Lett. 2003, 5, 6, 853-856.

(3) Hilborn, J. W.; MacKnight, E.; Pincock, J. A.; Wedge, P. J. J. Am. Chem. Soc. 1994, 116, 3337-3346.

(4) Barton, D. H. R.; Hervé, Y.; Potier, P.; Thierry, J. Tetrahedron 1988, 44, 17, 5479-5486.

(5) Sajiki, H.; Hirota, K. Tetrahedron 1998, 54, 46, 13981-13996. 
(6) Nguyen, D. L.; Seyer, R.; Heitz, A.; Castro, B. J. Chem. Soc. Perkin Trans. 1 1985, 10251032.

(7) Noguchi, T.; Hasegawa, M.; Tomisawa, K.; Mitsukuchi, M. Bioorg. Med. Chem. 2003, 11, $4729-4742$.

(8) Caddick, S.; Afonso, C. A. M.; Candeias, S. X.; Hitchcock, P. B.; Jenkins, K.; Murtagh, L.; Pardoe, D.; Santos, A. G.; Treweeke, N. R.; Weaving, R. Tetrahedron 2001, 57, 65896605.

(9) Mahboobi, S.; Popp, A.; Burgemeister, T.; Schollmeyer, D. Tetrahedron: Asymmetry 1998, 9, 2369-2376.

(10) Wang, W.; Xiong, C.; Yang, J.; Hruby, V. J. Synthesis 2002, 1, 28, 94-98.

(11) Sheehan, J. C.; Cruickshank, P. A.; Boshart, G. L. J. Org. Chem. 1961, 26, 2525-2528.

(12) Li, W.-R.; Chou, H.-H. Synthesis 2000, 1, 84-90.

(13) Zabrocki, J.; Dunbar Jr. J. B.; Marshall, K. W.; Toth, M. V.; Marshall, G. R. J. Org. Chem. 1992, 57, 202-209.

(14) Pierson, M. E.; Comstock, J. M.; Simmons, R. D.; Kaiser, F.; Julien, R.; Zongrone, J.; Rosamond, J. D. J. Med. Chem. 1997, 40, 4302-4307.

(15) Kokotos, G.; Six, D. A.; Loukas, V.; Smith, T.; Constantinou-Kokotou, V.; HadjipavlouLitina, D.; Kotsovolou, S.; Chiou, A.; Beltzner, C. C.; Dennis, E. A. J. Med. Chem. 2004, 47, $3615-3628$ 